FACTA UNIVERSITATIS (NIŠ)

Ser. Math. Inform. Vol. 36, No 2 (2021), 237-247

https://doi.org/10.22190/FUMI190313018R

Original Scientific Paper

\title{
FIXED POINT RESULTS IN COMPLEX VALUED METRIC SPACES WITH AN APPLICATION
}

\author{
Rashwan A. Rashwan ${ }^{1}$, Hasanen A. Hammad ${ }^{2}$ and Liliana Guran ${ }^{3}$ \\ 1 Assuit University, Faculty of Science, Department of Mathematics, \\ Assuit 71516, Egypt \\ 2 Sohag University, Faculty of Science, Department of Mathematics, \\ Sohag 82524, Egypt \\ 3 Vasile Goldiş Western University, Faculty of Farmacy, \\ Department of Pharmaceutical Sciences, 310045, Romania
}

\begin{abstract}
In this paper, we introduce fixed point theorem for a general contractive condition in complex valued metric spaces. Also, some important corollaries under this contractive condition are obtained. As an application, we find a unique solution for Urysohn integral equations and some illustrative examples are given to support our obtaining results. Our results extend and generalize the results of Azam et al. [2] and some other known results in the literature.

Key words: Single-valued mappings; complex valued metric spaces; common fixed point; nonlinear integral equations.
\end{abstract}

\section{Introduction}

A number of articles have been dedicated to the improvement and generalization of Banach contraction mapping principle. There exists various generalizations of the contraction principle, roughly obtained by weakening the contractive properties of the mapping and possibly, by simultaneously giving the space a sufficiently rich structure, in order to compensate the relaxation of the contractiveness, or by extending the structure of the space.

Received March 13, 2019; accepted: April 2, 2019

Communicated by Dragana Cvetković-Ilić

Corresponding Author: Hasanen A. Hammad, Sohag University, Faculty of Science, Department of Mathematics, Sohag 82524, Egypt | E-mail: h.elmagd89@gmail.com

2010 Mathematics Subject Classification. Primary 47H09; Secondary 47H10

(C) 2021 by University of Niš, Serbia | Creative Commons License: CC BY-NC-ND 
Also, several fixed point theorems are obtained by combining the two ways previously described or by adding supplementary conditions (see, for example, $[1$, $4,8,10,14,17,22])$.

The complex valued metric spaces is more general than ordinary metric spaces. According to this concept, a number of articles related to fixed point theory and it's application are presented (see, for example, [3, 5, 6, 7, 9, 11, 12, 13, 15, 16, 18, $19,20,21,23])$.

In this paper, we prove some fixed point theorem in complex valued metric spaces under contractive condition for single-valued mappings. Moreover, we give a result of existence and uniqueness for solutions of a nonlinear system of integral equations. Finally, we will give some explained examples to strengthen our results.

\section{Preliminaries}

In this section, we recall some known notations and definitions that will be used in the sequel.

Let $\mathbb{C}$ be the set of complex numbers and $z_{1}, z_{2} \in \mathbb{C}$. Define a partial order $\precsim$ on $\mathbb{C}$ as follows: $z_{1} z_{2}$ if and only if $\operatorname{Re}\left(z_{1}\right) \leq \operatorname{Re}\left(z_{2}\right)$ and $\operatorname{Im}\left(z_{1}\right) \leq \operatorname{Im}\left(z_{2}\right)$. It follows that $z_{1} \precsim z_{2}$ if one of the following conditions is satisfied:

$$
\begin{aligned}
& \left(C_{1}\right) \operatorname{Re}\left(z_{1}\right)=\operatorname{Re}\left(z_{2}\right) \text { and } \operatorname{Im}\left(z_{1}\right)<\operatorname{Im}\left(z_{2}\right), \\
& \left(C_{2}\right) \operatorname{Re}\left(z_{1}\right)<\operatorname{Re}\left(z_{2}\right) \text { and } \operatorname{Im}\left(z_{1}\right)=\operatorname{Im}\left(z_{2}\right), \\
& \left(C_{3}\right) \operatorname{Re}\left(z_{1}\right)<\operatorname{Re}\left(z_{2}\right) \text { and } \operatorname{Im}\left(z_{1}\right)<\operatorname{Im}\left(z_{2}\right), \\
& \left(C_{4}\right) \operatorname{Re}\left(z_{1}\right)=\operatorname{Re}\left(z_{2}\right) \text { and } \operatorname{Im}\left(z_{1}\right)=\operatorname{Im}\left(z_{2}\right),
\end{aligned}
$$

In particular, we write $z_{1} \lesssim z_{2}$ if $z_{1} \neq z_{2}$ and one of $\left(C_{1}\right),\left(C_{2}\right)$ and $\left(C_{3}\right)$ is satisfied and we write $z_{1} \prec z_{2}$ if only $\left(C_{3}\right)$ is satisfied.

Definition 2.1. [2] Let $X$ be a nonempty set. A mapping $d: X \times X \rightarrow \mathbb{C}$ is called a complex valued metric on $X$ if the following conditions holds for all $x, y, z \in X$,

$\left(C M_{1}\right) 0 \precsim d(x, y)$ and $d(x, y)=0$ if and only if $x=y$,

$\left(C M_{2}\right) d(x, y)=d(y, x)$,

$\left(C M_{3}\right) d(x, y) \precsim d(x, z)+d(z, y)$.

Then $d$ is called a complex valued metric on $X$ and $(X, d)$ is called a complex valued metric space.

For some examples of complex valued metric spaces (see $[2,5,12,18]$ ).

Definition 2.2. [2] Let $(X, d)$ be a complex valued metric space. Then

(i) A sequence $\left\{x_{n}\right\}$ in $X$ is said to be converged to $x \in X$ if for every $0 \prec \varepsilon \in \mathbb{C}$ there exists $N \in \mathbb{N}$ such that $d\left(x_{n}, x\right) \prec \varepsilon \forall n>N$. We denote this by $\lim _{n \rightarrow \infty} x_{n}=$ $x$ or $x_{n} \rightarrow x$ as $n \rightarrow \infty$. 
(ii) If for every $0 \prec \varepsilon \in \mathbb{C}$ there exists $N \in \mathbb{N}$ such that $d\left(x_{n}, x_{n+m}\right) \prec \varepsilon$ for all $n>N, m \in N$, Then $\left\{x_{n}\right\}$ is called a Cauchy sequence in $(X, d)$.

(iii) If every Cauchy sequence in $X$ is convergent in $X$ then $(X, d)$ is called a complete complex valued metric space.

Lemma 2.1. [2] Let $(X, d)$ be a complex valued metric space and $\left\{x_{n}\right\}$ be a sequence in $X$. Then $\left\{x_{n}\right\}$ converges to $x$ if and only if $\left|d\left(x_{n}, x\right)\right| \rightarrow 0$ as $n \rightarrow \infty$.

Lemma 2.2. [2] Let $(X, d)$ be a complex valued metric space. Then a sequence $\left\{x_{n}\right\}$ in $X$ is a Cauchy sequence if and only if $\left|d\left(x_{n}, x_{n+m}\right)\right| \rightarrow 0$ as $n \rightarrow \infty$, where $m \in N$.

\section{Main result}

We state and prove our first result.

Theorem 3.1. Let $(X, d)$ be a complete complex valued metric space and $S, T$ : $X \rightarrow X$ such that

$$
d(S x, T y) \precsim \alpha M(x, y),
$$

for all $x, y \in X$, where $0<\alpha<1$ and

$$
M(x, y)=\max \left\{d(x, y), \frac{d(x, S x) d(y, T y)}{1+d(x, y)}, \frac{d(x, T y) d(y, S x)}{1+d(x, y)}\right\} .
$$

Then there exists a unique common fixed point of the pair mappings $(S, T)$.

Proof. Let $x_{0}$ be arbitrary point in $X$ and define a sequence $\left\{x_{n}\right\}$ as follows:

$$
x_{2 n+1}=S x_{2 n} \text { and } x_{2 n+2}=T x_{2 n+1}, n=0,1,2, . .
$$

Then, by (3.1) and (3.2), we get

$$
\begin{aligned}
& d\left(x_{2 n+1}, x_{2 n+2}\right)=d\left(S x_{2 n}, T x_{2 n+1}\right) \\
& \precsim \alpha \max \left\{d\left(x_{2 n}, x_{2 n+1}\right), \frac{d\left(x_{2 n}, S x_{2 n}\right) d\left(x_{2 n+1}, T x_{2 n+1}\right)}{1+d\left(x_{2 n}, x_{2 n+1}\right)},\right. \\
& \left.\frac{d\left(x_{2 n}, T x_{2 n+1}\right) d\left(x_{2 n+1}, S x_{2 n}\right)}{1+d\left(x_{2 n}, x_{2 n+1}\right)}\right\} \\
& \precsim \alpha \max \left\{d\left(x_{2 n}, x_{2 n+1}\right), \frac{d\left(x_{2 n}, x_{2 n+1}\right) d\left(x_{2 n+1}, x_{2 n+2}\right)}{1+d\left(x_{2 n}, x_{2 n+1}\right)},\right. \\
& \left.\frac{d\left(x_{2 n}, x_{2 n+2}\right) d\left(x_{2 n+1}, x_{2 n+1}\right)}{1+d\left(x_{2 n}, x_{2 n+1}\right)}\right\} \\
& \precsim \alpha \max \left\{d\left(x_{2 n}, x_{2 n+1}\right), d\left(x_{2 n+1}, x_{2 n+2}\right)\right\} \text {. }
\end{aligned}
$$

If $\max \left\{d\left(x_{2 n}, x_{2 n+1}\right), d\left(x_{2 n+1}, x_{2 n+2}\right)\right\}=d\left(x_{2 n+1}, x_{2 n+2}\right)$, then

$$
d\left(x_{2 n+1}, x_{2 n+2}\right) \precsim \alpha d\left(x_{2 n+1}, x_{2 n+2}\right),
$$


This leads to, $\alpha \geq 1$, a contradiction. Therefore

$$
d\left(x_{2 n+1}, x_{2 n+2}\right) \precsim \alpha d\left(x_{2 n}, x_{2 n+1}\right) .
$$

Similarly, we can obtain that

$$
d\left(x_{2 n+2}, x_{2 n+3}\right) \precsim \alpha d\left(x_{2 n+1}, x_{2 n+2}\right) .
$$

From (3.3) and (3.4) for all $n=0,1,2, .$. , we can write

$$
d\left(x_{n+1}, x_{n+2}\right) \precsim \alpha d\left(x_{n}, x_{n+1}\right) \precsim \ldots \precsim \alpha^{n+1} d\left(x_{\circ}, x_{1}\right) .
$$

So for $m>n$,

$$
\begin{aligned}
d\left(x_{n}, x_{m}\right) & \precsim d\left(x_{n}, x_{n+1}\right)+d\left(x_{n+1}, x_{n+2}\right)+\ldots+d\left(x_{m-1}, x_{m}\right) \\
& \precsim\left(\alpha^{n}+\alpha^{n+1}+\ldots+\alpha^{m-1}\right) d\left(x_{\circ}, x_{1}\right) \\
& \precsim\left(\frac{\alpha^{n}}{1-\alpha}\right) d\left(x_{\circ}, x_{1}\right) .
\end{aligned}
$$

So,

$$
\left|d\left(x_{n}, x_{m}\right)\right| \precsim\left(\frac{\alpha^{n}}{1-\alpha}\right)\left|d\left(x_{\circ}, x_{1}\right)\right| \rightarrow 0 .
$$

As $n \rightarrow \infty$, therefore $\left\{x_{n}\right\}$ is a Cauchy sequence in $X$. Since $X$ is complete, then there exists $u \in X$ such that $x_{n} \rightarrow u$. If $S$ and $T$ are not continuous, it follows that $u=S u$, otherwise $d(u, S u)=z>0$ and we would then have

$$
\begin{aligned}
z & \precsim d\left(u, x_{2 k+2}\right)+d\left(S u, x_{2 k+2}\right) \\
& \precsim d\left(u, x_{2 k+2}\right)+d\left(S u, T x_{2 k+1}\right) \\
& \precsim d\left(u, x_{2 k+2}\right)+\alpha \max \left\{d\left(u, x_{2 k+1}\right), \frac{d(u, S u) d\left(x_{2 k+1}, T x_{2 k+1}\right)}{1+d\left(u, x_{2 k+1}\right)},\right. \\
& \left.\frac{d\left(u, T x_{2 k+1}\right) d\left(x_{2 k+1}, S u\right)}{1+d\left(u, x_{2 k+1}\right)}\right\} \\
& \precsim d\left(u, x_{2 k+2}\right)+\alpha \max \left\{d\left(u, x_{2 k+1}\right),\right. \\
& \left.\frac{d(u, S u) d\left(x_{2 k+1}, x_{2 k+2}\right)}{1+d\left(u, x_{2 k+1}\right)}, \frac{d\left(u, x_{2 k+2}\right) d\left(x_{2 k+1}, S u\right)}{1+d\left(u, x_{2 k+1}\right)}\right\} \\
& \precsim d\left(u, x_{2 k+2}\right)+\alpha \max \{0,0, z\} \\
& \precsim d\left(u, x_{2 k+2}\right)+\alpha z .
\end{aligned}
$$

This yields,

$$
|z| \leq\left|d\left(u, x_{2 k+2}\right)\right|+\alpha|z|
$$

That is $\alpha \geq 1$, a contradiction again and hence, $u=S u$. It follows similarly that $u=T u$.

If $S$ and $T$ are continuous, i.e., the continuity of $S$, yields

$$
u=\lim _{n \rightarrow \infty} x_{2 n+2}=\lim _{n \rightarrow \infty} S x_{2 n+1}=S \lim _{n \rightarrow \infty} x_{2 n+1}=S u .
$$


Similarly, $u=T u$. Hence the pair $(S, T)$ has a common fixed point.

For the uniqueness, assume that $v \in X$ is a second common fixed point of $S$ and $T$. Then

$$
\begin{aligned}
d(u, v) & =d(S u, T v) \\
& \precsim \alpha \max \left\{d(u, v), \frac{d(u, S u) d(v, T v)}{1+d(u, v)}, \frac{d(u, T v) d(v, S u)}{1+d(u, v)}\right\} \\
& \precsim \alpha d(u, v) .
\end{aligned}
$$

This implies that $u=v$, this completes the proof.

If we take $S=T$ in the above theorem we have we have the following immediate consequences.

Corollary 3.1. $(X, d)$ be a complete complex valued metric space and $S: X \rightarrow X$ satisfy

$$
d(S x, S y) \precsim \alpha M(x, y),
$$

for all $x, y \in X$, where $0<\alpha<1$ and

$$
M(x, y)=\max \left\{d(x, y), \frac{d(x, S x) d(y, S y)}{1+d(x, y)}, \frac{d(x, S y) d(y, S x)}{1+d(x, y)}\right\} .
$$

Then $S$ has a unique fixed point on $X$.

Corollary 3.2. Let $(X, d)$ be a complete complex valued metric space and $S: X \rightarrow$ $X$ satisfy

$$
d\left(S^{n} x, S^{n} y\right) \precsim \alpha M(x, y)
$$

for all $x, y \in X$, where $0<\alpha<1$ and

$$
M(x, y)=\max \left\{d(x, y), \frac{d\left(x, S^{n} x\right) d\left(y, S^{n} y\right)}{1+d(x, y)}, \frac{d\left(x, S^{n} y\right) d\left(y, S^{n} x\right)}{1+d(x, y)}\right\} .
$$

Then $S$ has a unique fixed point.

Proof. By Corollary 3.1, we obtain $v \in X$ such that

$$
S^{n} v=v .
$$

From the fact

$$
\begin{aligned}
d(S v, v) & =d\left(S S^{n} v, S^{n} v\right)=d\left(S^{n} S v, S^{n} v\right) \\
& \precsim \alpha \max \left\{d(S v, v), \frac{d\left(S v, S^{n} S v\right) d\left(v, S^{n} v\right)}{1+d(S v, v)}, \frac{d\left(S v, S^{n} v\right) d\left(v, S^{n} S v\right)}{1+d(S v, v)}\right\} \\
& \precsim \alpha \max \left\{d(S v, v), \frac{d\left(S v, S S^{n} v\right) d\left(v, S^{n} v\right)}{1+d(S v, v)}, \frac{d\left(S v, S^{n} v\right) d\left(v, S S^{n} v\right)}{1+d(S v, v)}\right\} \\
& =\alpha d(S v, v) .
\end{aligned}
$$

The result is follows. 


\section{An application to Urysohn integral type equations}

In this section, we apply Theorem 3.1 to prove the existence of a unique solution to the following Urysohn integral type equations:

$$
\left\{\begin{array}{l}
x(t)=h(t)+\int_{q}^{b} K_{1}(t, s, x(s)) d s \\
y(t)=h(t)+\int_{a}^{b} K_{2}(t, s, y(s)) d s
\end{array} .\right.
$$

where,

(i) $x(t)$ and $y(t)$ are unknown variables for each $t \in[a, b], a>0$,

(ii) $h(t)$ is the deterministic free term defined for $t \in[a, b]$,

(iii) $K_{1}(t, s)$ and $k_{2}(t, s)$ are deterministic kernels defined for $t, s \in[a, b]$. Let $X=\left(C[a, b], \mathbb{R}^{n}\right), a>0$ and $d: X \times X \rightarrow \mathbb{R}^{n}$ defined by

$$
d(x, y)=\sup _{t \in[a, b]}\|x(t)-y(t)\|_{\infty} \sqrt[3]{1+b^{3}} e^{i \cot ^{-1} b},
$$

for all $x, y \in X, i=\sqrt{-1} \in \mathbb{C}$.

It's obvious that $\left(C[a, b], \mathbb{R}^{n},\|\cdot\|_{\infty}\right)$ is a complete complex valued metric space.

Next, we consider a system (4.1) under the following conditions:

$\left(H_{1}\right) h(t) \in X$,

$\left(H_{2}\right) K_{1}, K_{2}:[a, b] \times[a, b] \times \mathbb{R}^{n} \rightarrow \mathbb{R}^{n}$ are continuous functions satisfying

$$
\left|K_{1}(t, s, u(s))-K_{1}(t, s, v(s))\right| \precsim \frac{1}{(b-a) e^{a b}} M(u, v),
$$

where,

$$
M(u, v)=\max \left\{d(u, v), \frac{d(u, S u) d(v, T v)}{1+d(u, v)}, \frac{d(u, T v) d(v, S u)}{1+d(u, v)}\right\} .
$$

Next, we state and prove the following theorem:

Theorem 4.1. $\left(C[a, b], \mathbb{R}^{n},\|\cdot\|_{\infty}\right)$ be a complete complex valued metric space, then the system (4.1) under the conditions $\left(H_{1}\right)$ and $\left(H_{2}\right)$ has a unique common solution.

Proof. For $x, y \in\left(C[a, b], \mathbb{R}^{n}\right)$ and $t \in[a, b]$, we define the continuous mappings $S, T: X \rightarrow X$ by

$$
\begin{aligned}
& S x(t)=h(t)+\int_{a}^{b} K_{1}(t, s, x(s)) d s, \\
& T y(t)=h(t)+\int_{a}^{b} K_{2}(t, s, y(s)) d s .
\end{aligned}
$$

By this, we have

$$
|S x(t)-T y(t)|=\int_{a}^{b}\left|K_{1}(t, s, x(s))-K_{2}(t, s, y(s))\right| d s
$$




$$
\begin{aligned}
& \precsim \int_{a}^{b} \frac{1}{(b-a) e^{a b}}|M(x, y)| d s \\
& =\frac{1}{(b-a) e^{a b}} \int_{a}^{b} \frac{e^{-i \cot ^{-1} b}}{\sqrt[3]{1+b^{3}}}|M(x, y)| \sqrt[3]{1+b^{3}} e^{i \cot ^{-1} b} d s \\
& \precsim \frac{1}{(b-a) e^{a b}} \frac{e^{-i \cot ^{-1} b}}{\sqrt[3]{1+b^{3}}}\|M(x, y)\|_{\infty} \int_{a}^{b} d s \\
& =\frac{1}{e^{a b}} \frac{e^{-i \cot ^{-1} b}}{\sqrt[3]{1+b^{3}}}\|M(x, y)\|_{\infty} .
\end{aligned}
$$

This gives,

$$
\sqrt[3]{1+b^{3}}|S x(t)-T y(t)| e^{-i \cot ^{-1} b} \precsim \frac{1}{e^{a b}}\|M(x, y)\|_{\infty},
$$

or, equivalently

$$
\|S x(t)-T y(t)\|_{\infty} \precsim \frac{1}{e^{a b}}\|M(x, y)\|_{\infty},
$$

or,

$$
d(S x, T y) \precsim \alpha M(x, y) .
$$

So, the condition (3.1) of Theorem 3.1 is satisfied with $0<\alpha=\frac{1}{e^{a b}}<1$, Therefore the system (4.1) has a unique common solution on $X$.

\section{Examples}

In this section we present some important examples to support our obtained results.

Example 5.1. Let $X=\mathbb{C}$ be a set of complex number. Define $d^{\prime}: \mathbb{C} \times \mathbb{C} \rightarrow \mathbb{C}$, by

$$
d^{\prime}\left(z_{1}, z_{2}\right)=d\left(x_{1}, x_{2}\right)+i d\left(y_{1}, y_{2}\right)
$$

for all $z_{1}, z_{2} \in \mathbb{C}$, where $z_{1}=x_{1}+i y_{1}=\left(x_{1}, y_{1}\right)$ and $z_{2}=x_{2}+i y_{2}=\left(x_{2}, y_{2}\right)$. If $(X, d)$ is a complex valued metric space, Then $\left(X, d^{\prime}\right)$ is too.

Example 5.2. Let $X=\mathbb{C}$ be a set of complex number. Define $d: \mathbb{C} \times \mathbb{C} \rightarrow \mathbb{C}$, by

$$
d\left(z_{1}, z_{2}\right)=\sqrt{\left(x_{1}-x_{2}\right)^{2}+i\left(y_{1}-y_{2}\right)^{2}}
$$

where $z_{1}=x_{1}+i y_{1}$ and $z_{2}=x_{2}+i y_{2}$. Then $(X, d)$ is a complex valued metric space.

Example 5.3. Let $X=[0, \infty)$ define the distance $d: X \times X \rightarrow \mathbb{C}$ by

$$
d(x, y)=i|x-y| .
$$


It's clearly $(X, d)$ is a complete complex valued metric space. We define the two selfmappings $S$ and $T$ as

$$
S x=2 x^{2}-1, T x=(2-x)^{2} .
$$

Then the contractive condition (3.1) is satisfied, indeed for $x=\frac{1}{3}$ and $y=3$, we can write by the simple calculations,

$$
d(S x, T y)=\frac{16}{9} i
$$

and

$$
M(x, y)=\max \left\{\frac{8}{3} i, \frac{-40}{3+8 i}, \frac{-68}{9(3+8 i)}\right\}=\frac{8}{3} i
$$

So,

$$
\frac{16 i}{9} \precsim \alpha \frac{8 i}{3}
$$

Therefore, the conditions of Theorem 3.1 are verified with $\alpha=\frac{2}{3}<1$ and $1 \in X$ is a unique common fixed point of $S$ and $T$.

Example 5.4. Let $X=[0, \infty)$ and $d: X \times X \rightarrow \mathbb{C}$ be a mapping defined by

$$
d(x, y)=|x-y|+i|x-y| .
$$

Clearly $(X, d)$ is a complete complex valued metric space. Define a self-mapping $S$ by

$$
S x=\frac{2}{\pi} \sin ^{-1} x .
$$

To verify the contractive condition of Corollary 3.1, we take $x=\frac{1}{2}$ and $y=\frac{\sqrt{3}}{2}$, one can write by the simple calculations,

$$
d(S x, S y) \simeq 0.1667(1+i)
$$

and

$$
M(x, y) \simeq \max \{0.3660(1+i), 0.0483 i, 0.1301 i\} \simeq 0.3660(1+i) .
$$

So,

$$
0.1667(1+i) \precsim \alpha 0.3660(1+i) .
$$

Therefore, all conditions of corollary 3.1 are satisfied with $\alpha \simeq 0.4555<1$ and $1 \in X$ is a unique fixed point of $S$.

Example 5.5. Let $X=C([0,2], \mathbb{R}), b>0$ and for every $x, y \in X$ let

$$
\begin{aligned}
N_{x y} & =\max _{t \in[0,2]}|x(t)-y(t)|, \\
d(x, y) & =N_{x y} \sqrt[3]{1+b^{3}} e^{i \cot ^{-1} b} .
\end{aligned}
$$


Define $S: X \rightarrow X$ by

$$
S x(t)=1+3 \int_{0}^{t} u^{2} x(u) d u, t \in[0,2] .
$$

For every $x, y \in X$, we have

$$
\begin{aligned}
d(S x, S y) & =N_{S x S y} \sqrt[3]{1+b^{3}} e^{i \cot ^{-1} b}=\max _{t \in[0,2]}|S x(t)-S y(t)| \sqrt[3]{1+b^{3}} e^{i \cot ^{-1} b} \\
& \precsim 3 \int_{0}^{2} \max _{t \in[0,2]}|x(u)-y(u)| u^{2} \sqrt[3]{1+b^{3}} e^{i \cot ^{-1} b} d u \\
& \precsim 8 d(x, y) .
\end{aligned}
$$

Similarly,

$$
d\left(S^{n} x, S^{n} y\right) \precsim \frac{8^{n}}{n !} d(x, y) \precsim \frac{8^{n}}{n !} M(x, y),
$$

where,

$$
\frac{8^{n}}{n !} \simeq \begin{cases}295.894 & \text { If } n=10 \\ 26.906 & \text { If } n=15 \\ 1.185 & \text { If } n=19 \\ 0.474 & \text { If } n=20\end{cases}
$$

Thus for $\alpha \simeq 0.474<1, n=20$, all conditions of Corollary 3.2 are satisfied and so $S$ has a unique fixed point, which is the unique solution of the integral equation:

$$
x(t)=1+3 \int_{0}^{t} u^{2} x(u) d u, t \in[0,2],
$$

or the differential equation (initial value problem):

$$
x^{\prime}(t)-3 x^{2} t=0, t \in[0,2], t(0)=1 .
$$

Example 5.6. Let $X=C([a, b], \mathbb{R})$ and the following nonlinear integral equation as the form:

$$
\left\{\begin{array}{l}
x(t)=e^{4 i t}+\int_{a}^{b}\left(\frac{e^{-\frac{1}{4}}}{4\left(t+\frac{i s}{1+i s}+x(s)\right)}\right) d s \\
y(t)=e^{4 i t}+\int_{a}^{b}\left(\frac{e^{-\frac{1}{4}}}{4\left(t+\frac{i s}{1+i s}+y(s)\right)}\right) d s
\end{array} .\right.
$$

System (5.1) is a particular case of system (4.1), where $h(t)=e^{4 i t}$ and

$$
K_{j}\left(t, s, u_{j}(s)\right)=\left(\frac{e^{-\frac{1}{4}}}{4\left(t+\frac{i s}{1+i s}+u_{j}(s)\right)}\right), j=1,2 .
$$


It's obvious that $\left(H_{1}\right)$ is satisfied, for $\left(H_{2}\right)$, we get

$$
\begin{aligned}
\left|K_{1}(t, s, x(s))-K_{2}(t, s, y(s))\right| & =\frac{1}{4} e^{-\frac{1}{4}}\left|\frac{x(s)-y(s)}{\left(t+\frac{i s}{1+i s}+x(s)\right)\left(t+\frac{i s}{1+i s}+y(s)\right)}\right| \\
& \precsim \frac{1}{4} e^{-\frac{1}{4}}|x(s)-y(s)| .
\end{aligned}
$$

Therefore, $\left(H_{2}\right)$ is hold with $\alpha=\frac{1}{4} e^{-\frac{1}{4}}<1$ and $M(x, y)=|x(s)-y(s)|$. By Theorem 4.1, the system (5.1) has a unique solution.

\section{REF EREN C ES}

1. I. Arandjelović, Z. Kadelburg and S. Radenović, Boyd-Wong-type common fixed point results in cone metric spaces, Appl. Math. Comput., 217 (2011), 7167-7171.

2. A. Azam, B. Fisher and M. Khan, Common fixed point theorems in complex valued metric spaces, Numerical Functional Analysis and Optimization, 32:3 (2011), 243-253.

3. S. Bhatt, S. Chaukiyal and R. C. Dimri, A common fixed point theorem for weakly compatible maps in complex valued metric spaces, Int. J. Math. Sci. Appl., 1(3) (2011), $1385-1389$.

4. D. W. Boyd and J. S. W. Wong, On nonlinear contractions, Proc. Amer. Math. Soc., 20 (1969), 458-464.

5. S. ChANDOK and D. Kumar, Some common fixed point results for rational type contraction mappings in complex valued metric spaces, J. Oper., 2013 (2013), 1-7.

6. S. K. DATtA and S. Ali, A common fixed point theorem under contractive condition in complex valued metric spaces, Int. J. Advanced Scientific and Technical Research, 2(6) (2012), 467-475.

7. A. K. DuBEY, Complex valued b-metric spaces and common fixed point theorems under rational contractions, J. Complex Anal., 2016 (2016), 1-7.

8. J. Dugundji and A. Granas, Fixed Point Theory, Monograe Matematycne, Warsazawa, (1982).

9. S. U. Khan, M. Arashad, H. K. Nashine and M. Nazam, Some common fixed points of generalized contractive mappings on complex valued metric spaces, J. Ana. Num. Theor., 5(1) (2017), 73-80.

10. W. A. KIRK and B. Sems, Handbook of Metric Fixed Point Theory, Kluwer Academic Publishers, Iowa City and Newcastle, (2001).

11. S. MANRo, Some common fixed point theorems in complex valued metric space using implicit function, Int. J. Anal. Appl., 2(1) (2013), 62-70.

12. T. Mitra, A common coupled fixed point result in complex valued metric space for two mappings, Int. J. Current. Res., 7(8) (2015), 19555-19559.

13. H. K. NAShine, M. Imdad and M. HASSAn, Common fixed point theorems under rational contractions in complex valued metric spaces, J. Nonlin. Sci. Appl., 7 (2014), 42-50.

14. E. Rakотсн, A note in contractive mappings, Proc. Amer. Math. Soc., 13 (1962), 459-465. 
15. R. A. Rashwan, H. A. Hammad and M. G. Mahmoud, Common fixed point theorems in complex-valued $S$-metric spaces via implicit relations with applications, Results in Fixed Point Theory and Applications, Volume 2019, Article ID 2018034, 17 pages.

16. F. RouzkARD and M. IMDAD, Some common fixed point theorems complex valued metric spaces, Comput. Math. Appl., 64 (6) (2012), 1866-1874.

17. I. A. Rus, Generalized Contractions and Applications, Cluj University Press, ClujNapoca, (2001).

18. N. Singh, D. Singh, A. BAdAL and V. Joshi, Fixed point theorems in complex valued metric spaces, J. Egyptian Math. Soc., 24 (2016), 402-409.

19. W. Sintunavarat and P. Kumam, Generalized common fixed point theorems in complex valued metric spaces and applications, J. Inequality Appl., (1) (2012), 1-12.

20. W. Sintunavarat, J. C. YeOL and P. Kumam, Urysohn integral equations approach by common fixed points in complex valued metric spaces, Adv. Diff. Equ., 2013 (2013), $1-14$.

21. K. Sitthikul and S. Saejung, Some fixed point theorems in complex valued metric space, Fixed Point Theory Appl., (1) (2012), 1-11.

22. E. TARAFDAR, An approach to fixed-point theorems on uniform spaces, Trans. Amer. Math. Soc., 191 (1974), 209-225.

23. R. K. Verma and H. K. Pathak, Common fixed point theorems using property (E.A.) in complex valued metric spaces, Thai J. Math., 11 (2) (2013), 347-355. 